\title{
Hyperbolically convex functions
}

\author{
by Wancang Ma and David Minda (Cincinnati, Ohio)
}

\begin{abstract}
We investigate univalent holomorphic functions $f$ defined on the unit disk $\mathbb{D}$ such that $f(\mathbb{D})$ is a hyperbolically convex subset of $\mathbb{D}$; there are a number of analogies with the classical theory of (euclidean) convex univalent functions. A subregion $\Omega$ of $\mathbb{D}$ is called hyperbolically convex (relative to hyperbolic geometry on $\mathbb{D}$ ) if for all points $a, b$ in $\Omega$ the arc of the hyperbolic geodesic in $\mathbb{D}$ connecting $a$ and $b$ (the arc of the circle joining $a$ and $b$ which is orthogonal to the unit circle) lies in $\Omega$. We give several analytic characterizations of hyperbolically convex functions. These analytic results lead to a number of sharp consequences, including covering, growth and distortion theorems and the precise upper bound on $\left|f^{\prime \prime}(0)\right|$ for normalized $\left(f(0)=0\right.$ and $\left.f^{\prime}(0)>0\right)$ hyperbolically convex functions. In addition, we find the radius of hyperbolic convexity for normalized univalent functions mapping $\mathbb{D}$ into itself. Finally, we suggest an alternate definition of "hyperbolic linear invariance" for locally univalent functions $f: \mathbb{D} \rightarrow \mathbb{D}$ that parallels earlier definitions of euclidean and spherical linear invariance.
\end{abstract}

1. Introduction. Our principal goal is to develop the theory of hyperbolically convex functions on the unit disk $\mathbb{D}$. A holomorphic, univalent function $f$ defined on $\mathbb{D}$ is called hyperbolically convex if the image region $f(\mathbb{D})$ is a hyperbolically convex subset of $\mathbb{D}$. This is a subclass of the set of all univalent functions mapping $\mathbb{D}$ into itself. The classical theory of euclidean convex univalent functions defined on the unit disk is well developed; for example, see [1] or [3]. The theory of spherically convex univalent functions has received less attention, but some basic results are known; see [5], [8] and [11].

For $\alpha>0$ let $K_{h}(\alpha)$ denote the family of all holomorphic functions $f: \mathbb{D} \rightarrow \mathbb{D}$ such that $f$ is univalent, $f(0)=0, f^{\prime}(0)=\alpha$, and $f(\mathbb{D})$ is a hyperbolically convex region in $\mathbb{D}$. Schwarz' Lemma implies that $\alpha \leq 1$ and $\alpha=1$ only when $f$ is the identity. We first determine the Koebe region $\bigcap\left\{f(\mathbb{D}): f \in K_{h}(\alpha)\right\}$ for the family $K_{h}(\alpha)$. Then we obtain a number of

1991 Mathematics Subject Classification: 30C45, 30C25, 30C50.

Key words and phrases: hyperbolic convexity, distortion theorem, growth thoerem, linear invariance.

Research partially supported by a National Science Foundation Grant. 
characterizations of hyperbolically convex functions, including a connection with euclidean starlike functions. The connection with euclidean starlike functions is based upon the observation that a hyperbolically convex region containing the origin is starlike with respect to the origin. A computational technique is used to derive the sharp upper bound on $\left|f^{\prime \prime}(0)\right|$ over the family $K_{h}(\alpha)$. This leads to sharp growth and distortion theorems for $K_{h}(\alpha)$.

For $\alpha>0$, let $S B(\alpha)$ denote the family of holomorphic, univalent functions $f$ defined on $\mathbb{D}$ which satisfy $f(0)=0, f^{\prime}(0)=\alpha$ and $f(\mathbb{D}) \subset \mathbb{D}$. Of course, $\alpha \in(0,1]$ and $K_{h}(\alpha)$ is a subfamily of $S B(\alpha)$. It is natural to determine the radius of hyperbolic convexity for the family $S B(\alpha)$. In fact, we are able to find the radius of hyperbolic $k$-convexity for $S B(\alpha)$ for all $k \in[0,4]$; the value for $k=0$ corresponds to the radius of hyperbolic convexity.

Euclidean (spherical) convexity plays a fundamental role in euclidean (spherical) linear invariance ([6], [8]). This suggests that hyperbolic convexity should play a similar role relative to an appropriate notion of hyperbolic linear invariance. Our theory of hyperbolically convex functions enables us to show that there is a corresponding notion of hyperbolic linear invariance which exhibits strong similarities with both euclidean and spherical linear invariance. A related, but different, notion of hyperbolic linear invariance was given in [7]. This earlier definition of hyperbolic linear invariance was motivated by the desire to generalize certain aspects of the classical theory of bounded univalent functions in the same way the definition of euclidean linear invariance by Pommerenke [14] extended a number of results from univalent function theory.

2. Preliminaries. The hyperbolic metric on the unit disk $\mathbb{D}=\{z$ : $|z|<1\}$ is given by $\lambda_{\mathbb{D}}(z)|d z|=|d z| /\left(1-|z|^{2}\right)$. This metric is normalized to have Gaussian curvature -4 . Let $\Omega$ be a hyperbolic region in the complex plane $\mathbb{C}$; that is, $\mathbb{C} \backslash \Omega$ contains at least two points. The hyperbolic metric $\lambda_{\Omega}(z)|d z|$ on $\Omega$ is determined from $\lambda_{\Omega}(\varphi(z))\left|\varphi^{\prime}(z)\right|=\lambda_{\mathbb{D}}(z)$, where $\varphi$ is any holomorphic covering projection of $\mathbb{D}$ onto $\Omega$. This definition is independent of the choice of the holomorphic covering projection. When $\Omega$ is simply connected, a holomorphic covering projection of $\mathbb{D}$ onto $\Omega$ is a conformal mapping. The hyperbolic metric on any hyperbolic region $\Omega$ has Gaussian curvature -4 ; this means that

$$
\kappa\left(z, \lambda_{\Omega}\right)=-\frac{\Delta \log \lambda_{\Omega}(z)}{\lambda_{\Omega}^{2}(z)}=-4 .
$$

The hyperbolic distance between $a, b \in \Omega$ is defined by

$$
d_{\Omega}(a, b)=\inf \int_{\delta} \lambda_{\Omega}(z)|d z|
$$


where the infimum is taken over all rectifiable paths $\delta$ in $\Omega$ joining $a$ and $b$. A path $\gamma$ connecting $a$ and $b$ is called a hyperbolic geodesic if

$$
d_{\Omega}(a, b)=\int_{\gamma} \lambda_{\Omega}(z)|d z|
$$

Hyperbolic geodesics always exist, but need not be unique unless $\Omega$ is simply connected. The hyperbolic disk with center $a$ and hyperbolic radius $\varrho$ is $D_{\Omega}(a, \varrho)=\left\{z \in \Omega: d_{\Omega}(a, z)<\varrho\right\}$. For the unit disk $\mathbb{D}$,

$$
d_{\mathbb{D}}(a, b)=\frac{1}{2} \log \frac{1+\left|\frac{a-b}{1-\bar{a} b}\right|}{1-\left|\frac{a-b}{1-\bar{a} b}\right|}=\operatorname{artanh}\left|\frac{a-b}{1-\bar{a} b}\right| .
$$

The hyperbolic distance on $\mathbb{D}$ is invariant under Aut $(\mathbb{D})$, the group of conformal automorphisms of $\mathbb{D}$. The unique hyperbolic geodesic in $\mathbb{D}$ connecting $a$ and $b$ is the arc $\gamma$ of the circle through $a$ and $b$ that is orthogonal to the unit circle.

We define two differential operators for holomorphic functions $f: \mathbb{D} \rightarrow \mathbb{D}$. Set

and

$$
D_{h 1} f(z)=\frac{\left(1-|z|^{2}\right) f^{\prime}(z)}{1-|f(z)|^{2}}
$$

$$
D_{h 2} f(z)=\frac{\left(1-|z|^{2}\right)^{2} f^{\prime \prime}(z)}{1-|f(z)|^{2}}+\frac{2\left(1-|z|^{2}\right)^{2} \overline{f(z)} f^{\prime}(z)^{2}}{\left(1-|f(z)|^{2}\right)^{2}}-\frac{2 \bar{z}\left(1-|z|^{2}\right) f^{\prime}(z)}{1-|f(z)|^{2}} \text {. }
$$

The use of these two differential operators simplifies many formulas. For more information about these two operators, see [7]. In particular, we note the invariance property $\left|D_{h j}(R \circ f \circ S)\right|=\left|D_{h j} f\right| \circ S(j=1,2)$ which holds for all $R, S \in \operatorname{Aut}(\mathbb{D})$. Also, if $f(0)=0$, then $D_{h 1} f(0)=f^{\prime}(0)$ and $D_{h 2} f(0)=f^{\prime \prime}(0)$.

We shall make use of the notion of the (signed) hyperbolic curvature of a path; this is the geodesic curvature of a path relative to the hyperbolic metric. Suppose $\gamma: z=z(t), t \in I$, where $I$ is an interval on the real line, is a $C^{2}$ curve in a hyperbolic region $\Omega$ with nonvanishing tangent. The (signed) hyperbolic curvature $\kappa_{\Omega}(z, \gamma)$ of $\gamma$ at $z=z(t)$ is

$$
\begin{aligned}
\kappa_{\Omega}(z, \gamma) \lambda_{\Omega}(z) & =\kappa_{\mathrm{e}}(z, \gamma)-\frac{\partial \log \lambda_{\Omega}(z)}{\partial n} \\
& =\kappa_{\mathrm{e}}(z, \gamma)+2 \operatorname{Im}\left\{\frac{\partial \log \lambda_{\Omega}(z)}{\partial z} \cdot \frac{z^{\prime}(t)}{\left|z^{\prime}(t)\right|}\right\},
\end{aligned}
$$

where $\kappa_{\mathrm{e}}(z, \gamma)$ is the euclidean curvature of $\gamma$ at $z$ and $n=n(z)$ is the unit normal at $z$ which makes an angle $+\pi / 2$ with the tangent vector to $\gamma$ at $z$. 
In particular, if $\Omega=\mathbb{D}$, then

$$
\kappa_{\mathbb{D}}(z, \gamma)=\left(1-|z|^{2}\right) \kappa_{\mathrm{e}}(z, \gamma)+2 \operatorname{Im}\left\{\frac{\overline{z(t)} z^{\prime}(t)}{\left|z^{\prime}(t)\right|}\right\} .
$$

The hyperbolic curvature $\kappa_{\mathbb{D}}(0, \gamma)$ relative to $\mathbb{D}$ and the euclidean curvature $\kappa_{\mathrm{e}}(0, \gamma)$ coincide at the origin. Hyperbolic curvature on $\mathbb{D}$ is invariant un$\operatorname{der} \operatorname{Aut}(\mathbb{D})$; that is, $\kappa_{\mathbb{D}}(S(z), S \circ \gamma)=\kappa_{\mathbb{D}}(z, \gamma)$ for any $S \in \operatorname{Aut}(\mathbb{D})$. In $\mathbb{D}$ the arcs of constant hyperbolic curvature are subarcs of (euclidean) circular arcs; hyperbolic geodesics have hyperbolic curvature zero. For a locally univalent function $f: \mathbb{D} \rightarrow \mathbb{D}$ there is a simple formula relating the hyperbolic curvature of the paths $\gamma: z=z(t)$ and $f \circ \gamma[7]$ :

$$
\kappa_{\mathbb{D}}(f(z), f \circ \gamma)\left|D_{h 1} f(z)\right|=\kappa_{\mathbb{D}}(z, \gamma)+\operatorname{Im}\left\{\frac{D_{h 2} f(z)}{D_{h 1} f(z)} \cdot \frac{z^{\prime}(t)}{\left|z^{\prime}(t)\right|}\right\} .
$$

We briefly recall the notion of hyperbolic $k$-convexity; see [2] and [9] for additional information. A region $\Omega \subset \mathbb{D}$ is called hyperbolically $k$-convex $(k>0)$ relative to hyperbolic geometry on $\mathbb{D}$ if for any pair of distinct points $a, b \in \Omega$ the two shortest arcs of constant hyperbolic curvature $k$ connecting $a$ and $b$ lie in $\Omega$. A region $\Omega$ is hyperbolically convex if for all $a, b \in \Omega$ the hyperbolic geodesic connecting $a$ and $b$ lies in $\Omega$. Note that if $\Omega$ is hyperbolically convex and $0 \in \Omega$, then $\Omega$ is starlike with respect to the origin since hyperbolic geodesics in $\mathbb{D}$ through the origin are euclidean line segments. A hyperbolically convex region may be regarded as a hyperbolically 0 -convex region. The concept of hyperbolic $k$-convexity is invariant under $\operatorname{Aut}(\mathbb{D})$. A univalent function $f$ mapping $\mathbb{D}$ into itself is called hyperbolically $k$-convex if the image region $f(\mathbb{D})$ is hyperbolically $k$-convex. Hyperbolically $k$-convex functions were studied in [5] and [9].

It is known (see [2] and [9]) that $\Omega \subset \mathbb{D}$ is hyperbolically $k$-convex if $\partial \Omega$ is a Jordan curve of class $C^{2}$ and $\kappa_{\mathbb{D}}(z, \partial \Omega) \geq k$ for all $z \in \partial \Omega$. On the other hand, for $k=2$ and 4 , Flinn and Osgood [2] showed that if $\Omega \subset \mathbb{D}$ is hyperbolically $k$-convex with $C^{2}$ boundary then $\kappa_{\mathbb{D}}(z, \partial \Omega) \geq k$ for all $z \in \partial \Omega$. We now extend this result to all $k \geq 0$.

THEOREM 1. Let $\Omega \subset \mathbb{D}$ be a hyperbolically $k$-convex region with $C^{2}$ boundary. Then $\kappa_{\mathbb{D}}(z, \partial \Omega) \geq k$ for all $z \in \partial \Omega \cap \mathbb{D}$.

P r o of. Since both hyperbolic curvature and hyperbolic $k$-convexity are invariant under the group Aut $(\mathbb{D})$, it is enough to show that $\kappa_{\mathbb{D}}(0, \partial \Omega) \geq k$ if $0 \in \partial \Omega$.

First, we consider the case $k>0$. Assume that $\kappa_{\mathbb{D}}(0, \partial \Omega)<k$. Then $\kappa_{\mathrm{e}}(0, \partial \Omega)=\kappa_{\mathbb{D}}(0, \partial \Omega)<k$. Let $L$ be the circular arc tangent to $\partial \Omega$ at 0 with euclidean curvature $k$ such that $\Omega \cap\{z:|z|<\varepsilon\}$ lies inside $L$ for sufficiently small $\varepsilon>0$. Let $\Delta$ be the region in $\mathbb{D}$ outside $L$. Since $\kappa_{\mathrm{e}}(0, \gamma)<k$, there are two points $a$ and $b$ in $\mathbb{D}$ lying in different components of $\Omega \cap \Delta$. One 
of the curves connecting $a$ and $b$ with constant hyperbolic curvature $k$ lies entirely in $\bar{\Delta}$, so it cannot lie entirely in $\Omega$. This contradicts the hyperbolic $k$-convexity of $\Omega$, so we must have $\kappa_{\mathbb{D}}(0, \partial \Omega) \geq k$.

When $k=0$, we replace $L$ by the straight line tangent to $\partial \Omega$ at 0 and use a similar argument to that employed above.

3. Characterizations of hyperbolically convex functions. In [5] a transformation method was used to obtain a number of sharp growth and distortion theorems for hyperbolically $k$-convex functions when $k \geq 2$. For $0 \leq k<2$, this transformation method does not seem applicable and the corresponding problems for hyperbolically $k$-convex functions when $k \in$ $[0,2)$ remain open. In this section, we use a different approach to investigate hyperbolically convex functions.

We present an example of a hyperbolically convex function which plays an important role in the class $K_{h}(\alpha)$.

ExAmple 1 . For $0<\alpha \leq 1$, let $\gamma$ be the hyperbolic geodesic in $\mathbb{D}$ which is symmetric about the real axis and passes through $-\alpha /\left(1+\sqrt{1-\alpha^{2}}\right)$. The geodesic $\gamma$ determines two hyperbolic half-planes; let $\Omega$ be the one that contains the origin. Elementary computation shows that

$$
g_{\alpha}(w)=\frac{w(1+\alpha w)}{\alpha+w}
$$

maps $\Omega$ conformally onto $\mathbb{D}$ with $g_{\alpha}(0)=0$ and $g_{\alpha}^{\prime}(0)=1 / \alpha$. If $k_{\alpha}=g_{\alpha}^{-1}$, then $k_{\alpha}$ maps $\mathbb{D}$ conformally onto the hyperbolic half-plane $\Omega$ with $k_{\alpha}(0)=0$ and $k_{\alpha}^{\prime}(0)=\alpha$, so $k_{\alpha} \in K_{h}(\alpha)$. The function $k_{\alpha}$ has the explicit expression

$$
k_{\alpha}(z)=\frac{2 \alpha z}{1-z+\sqrt{1+2\left(2 \alpha^{2}-1\right) z+z^{2}}}
$$

and the power series expansion

$$
k_{\alpha}(z)=\alpha z+\alpha\left(1-\alpha^{2}\right) z^{2}+\alpha\left(1-\alpha^{2}\right)\left(1-2 \alpha^{2}\right) z^{3}+\ldots
$$

Now we give a different proof of the covering theorem for hyperbolically convex functions, which was obtained earlier by Mejia and Minda [9]. More precisely, they obtained the covering theorem for hyperbolically $k$-convex functions for all $k \geq 0$. For $0 \leq k<2$ they determined the radius of the disk covered by all normalized hyperbolically $k$-convex functions as the root of a complicated equation. Here we show that the covering theorem for the case $k=0$ is explicit and simple, which will be used to give a preliminary upper bound on the second coefficient of hyperbolically convex functions. A similar proof also works when $0<k<2$. 
THEOREM 2. Let $f \in K_{h}(\alpha)$. Then either $f(\mathbb{D})$ contains the closed disk

$$
\left\{w:|w| \leq \frac{\alpha}{1+\sqrt{1-\alpha^{2}}}\right\}
$$

or else $f$ is a rotation of $k_{\alpha}$, that is, $f(z)=e^{i \theta} k_{\alpha}\left(e^{-i \theta} z\right)$ for some real $\theta$.

Pr o of. Let $c$ be a point in $\mathbb{D} \backslash f(\mathbb{D})$ which is closest to the origin. Then

$$
F(z)=\frac{f(z)-c}{1-\bar{c} f(z)}
$$

is also hyperbolically convex and $0 \notin F(\mathbb{D})$. So $F(\mathbb{D})$ must lie in some half-disk of $\mathbb{D}$ which has a diameter $L^{\prime}$ as part of its boundary. Let $\Omega$ be the hyperbolic half-plane containing the origin and bounded by the image $L=T\left(L^{\prime}\right)$ of $L^{\prime}$ under the conformal automorphism $T(z)=(z+c) /(1+\bar{c} z)$. Then $f(\mathbb{D}) \subset \Omega$. Note that $c \in L \subset \mathbb{D} \backslash f(\mathbb{D})$ and $c$ is a closest point to the origin in $\mathbb{D} \backslash f(\mathbb{D})$, so $L$ must be symmetric with respect to the straight line passing through 0 and $c$. We may assume $c<0$ by rotating $f$ if necessary; then $L$ is symmetric with respect to the real axis. Consider the function $k_{\beta}$ of Example 1, where $\beta$ is determined from

$$
\frac{\beta}{1+\sqrt{1-\beta^{2}}}=-c .
$$

Define $g$ by $f(z)=k_{\beta}(g(z))$; then $g$ is holomorphic and univalent in $\mathbb{D}$ with $g(0)=0$ and $g(\mathbb{D}) \subset \mathbb{D}$. In other words, $f$ is subordinate to $k_{\beta}$; this implies that $\alpha \leq \beta$. Therefore,

$$
|c|=\frac{\beta}{1+\sqrt{1-\beta^{2}}} \geq \frac{\alpha}{1+\sqrt{1-\alpha^{2}}} .
$$

If $|c|=\alpha /\left(1+\sqrt{1-\alpha^{2}}\right)$, then $\alpha=\beta, g^{\prime}(0)=1, g(z)=z$ and so $f=k_{\alpha}$. This completes the proof.

Lemma 1. Suppose $\Omega \subset \mathbb{D}$ is hyperbolically convex in $\mathbb{D}$. Then for $c \in$ $\bar{\Omega} \cap \mathbb{D}$ and $\varrho>0, \Omega \cap D_{\mathbb{D}}(c, \varrho)$ is hyperbolically convex in $\Omega$.

P r o of. Since the hyperbolic distance and hyperbolic convexity are both invariant under Aut $(\mathbb{D})$, we may assume that $c=0$. Now we prove that the set $\Omega \cap D_{\mathbb{D}}(0, \varrho)=\Omega \cap\{z:|z|<\tanh (\varrho)\}$ is hyperbolically convex in $\Omega$. For each $z \in \Omega$, the half-open line segment $(0, z]$ lies in $\Omega$ because $\Omega$ is hyperbolically convex. Consequently, $\Omega$ is starlike with respect to 0 . By Proposition 2 of [10], we see that $\Omega \cap\{z:|z|<\tanh (\varrho)\}$ is hyperbolically convex in $\Omega$.

TheOREM 3. Suppose $f$ is holomorphic and locally univalent in $\mathbb{D}$ with $f(\mathbb{D}) \subset \mathbb{D}$. Then the following are equivalent.

(i) $f$ is hyperbolically convex. 
(ii) For $z \in \mathbb{D}$,

(iii) For $z \in \mathbb{D}$,

$$
\left|\frac{D_{h 2} f(z)}{2 D_{h 1} f(z)}\right|<1
$$

$$
\operatorname{Re}\left\{\frac{z f^{\prime \prime}(z)}{f^{\prime}(z)}+1+\frac{2 z \overline{f(z)} f^{\prime}(z)}{1-|f(z)|^{2}}\right\}>0 .
$$

Proof. (i) $\Rightarrow($ ii). Because hyperbolic convexity and the quantity $\left|D_{h 2} f(z) /\left(2 D_{h 1} f(z)\right)\right|$ are both invariant under Aut $(\mathbb{D})$, we need only establish the implication in the case when $z=0$ and $f(0)=0$. In this case we need to demonstrate that

$$
\left|\frac{f^{\prime \prime}(0)}{2 f^{\prime}(0)}\right|<1
$$

Let $\Omega=f(\mathbb{D})$ and $\beta>0$ be the radius of the largest open euclidean disk centered at 0 that is contained in $f(\mathbb{D})$. Then $\lambda_{\Omega}(f(z))\left|f^{\prime}(z)\right|=1 /\left(1-|z|^{2}\right)$ and formula (1) yield

$$
\lambda_{\Omega}(0) \kappa_{\Omega}(0, \gamma)=\kappa_{\mathrm{e}}(0, \gamma)-\operatorname{Im}\left\{\frac{f^{\prime \prime}(0)}{f^{\prime}(0)^{2}} \cdot \frac{w^{\prime}(0)}{\left|w^{\prime}(0)\right|}\right\}
$$

for a smooth curve $\gamma: w=w(t)$ with $w(0)=0$. As $\{w:|w| \leq \beta\} \subset \bar{\Omega}$, Lemma 1 implies that $\Omega \cap D_{\mathbb{D}}\left(\beta e^{i \theta}, \operatorname{artanh}(\beta)\right)$ is hyperbolically convex in $\Omega$ for all real $\theta$. Note that

$$
D_{\mathbb{D}}\left(\beta e^{i \theta}, \operatorname{artanh}(\beta)\right)=\left\{w:\left|\frac{w-\beta e^{i \theta}}{1-\beta e^{-i \theta} w}\right|<\beta\right\}
$$

is a euclidean disk with radius $\beta /\left(1+\beta^{2}\right)$ and has the origin as a boundary point. Let $\gamma_{\theta}: w=w(t)$, with $w(0)=0$, be a parametrization of the part of the boundary of this disk that lies in $\Omega$. Then $\kappa_{\mathrm{e}}\left(0, \gamma_{\theta}\right)=\beta+1 / \beta$. From Theorem 1 we deduce that $\kappa_{\Omega}\left(0, \gamma_{\theta}\right) \geq 0$ for all real $\theta$. We may now conclude that

$$
\left|\frac{f^{\prime \prime}(0)}{f^{\prime}(0)^{2}}\right| \leq \beta+\frac{1}{\beta}
$$

Set $\alpha=\left|f^{\prime}(0)\right|$. If $f$ is not a rotation of the function $k_{\alpha}$ given in Example 1 , then we know from Theorem 2 that $1>\beta>\alpha /\left(1+\sqrt{1-\alpha^{2}}\right)$. This yields

$$
\left|\frac{f^{\prime \prime}(0)}{2 f^{\prime}(0)}\right| \leq \frac{\alpha}{2}\left(\beta+\frac{1}{\beta}\right)<1
$$

On the other hand, if $f$ is a rotation of $k_{\alpha}$, then

$$
\left|\frac{f^{\prime \prime}(0)}{2 f^{\prime}(0)}\right|=1-\alpha^{2}<1 \text {. }
$$

This shows that $(\mathrm{i}) \Rightarrow(\mathrm{ii})$. 
(ii) $\Rightarrow$ (iii). Condition (ii) means that

or

$$
\left|\frac{\left(1-|z|^{2}\right) f^{\prime \prime}(z)}{f^{\prime}(z)}+\frac{2\left(1-|z|^{2}\right) \overline{f(z)} f^{\prime}(z)}{1-|f(z)|^{2}}-2 \bar{z}\right|<2,
$$

This yields

$$
\left|\frac{z f^{\prime \prime}(z)}{f^{\prime}(z)}+1+\frac{2 z \overline{f(z)} f^{\prime}(z)}{1-|f(z)|^{2}}-\frac{1+|z|^{2}}{1-|z|^{2}}\right|<\frac{2|z|}{1-|z|^{2}} .
$$

$$
\operatorname{Re}\left\{\frac{z f^{\prime \prime}(z)}{f^{\prime}(z)}+1+\frac{2 z \overline{f(z)} f^{\prime}(z)}{1-|f(z)|^{2}}\right\}>\frac{1-|z|}{1+|z|}>0
$$

(iii) $\Rightarrow$ (i). We start by showing that the image under $f$ of every circle centered at the origin has positive hyperbolic curvature. For $0<r<1$, consider the euclidean disk $D(0, r)$, with center 0 and radius $r$ and its positively oriented boundary $\gamma: z(t)=r e^{i t}, 0 \leq t \leq 2 \pi$. We know [7] that $\kappa_{\mathbb{D}}(z, \gamma)=r+r^{-1}>2$ and from formula (2) we get

$$
\begin{aligned}
\kappa_{\mathbb{D}}(f(z), f \circ \gamma)\left|D_{h 1} f(z)\right| \\
=\kappa_{\mathbb{D}}(z, \gamma)+\operatorname{Im}\left\{\frac{D_{h 2} f(z)}{D_{h 1} f(z)} \cdot \frac{i z}{r}\right\}=\kappa_{\mathbb{D}}(z, \gamma)+\operatorname{Re}\left\{\frac{D_{h 2} f(z)}{D_{h 1} f(z)} \cdot \frac{z}{r}\right\} \\
=\frac{1}{r}\left[r^{2}+1+\operatorname{Re}\left\{\frac{\left(1-|z|^{2}\right) z f^{\prime \prime}(z)}{f^{\prime}(z)}+\frac{2\left(1-|z|^{2}\right) z \overline{f(z)} f^{\prime}(z)}{1-|f(z)|^{2}}-2|z|^{2}\right\}\right] \\
=\frac{1}{r}\left[r^{2}+1+\left(1-r^{2}\right) \operatorname{Re}\left\{\frac{z f^{\prime \prime}(z)}{f^{\prime}(z)}+1+\frac{2 z \overline{f(z)} f^{\prime}(z)}{1-|f(z)|^{2}}-\frac{1+|z|^{2}}{1-|z|^{2}}\right\}\right] .
\end{aligned}
$$

By making use of (iii) we see that $\kappa_{\mathbb{D}}(f(z), f \circ \gamma)>0$.

Next we show that $f$ is univalent in $\mathbb{D}$. We may assume that $f(0)=$ 0 . If not, replace $f$ by the function $F(z)=(f(z)-f(0)) /(1-\overline{f(0)} f(z))$ which also has the property that $F \circ \gamma$ has positive hyperbolic curvature for $0<r<1$ since hyperbolic curvature is invariant under Aut $(\mathbb{D})$. Let $R=\sup \{r: f$ is univalent in $D(0, r)\}$. Note that $R>0$ because $f$ is locally univalent. If $R=1$, we are done. Suppose $0<R<1$. Then $f(D(0, R))$ is hyperbolically convex because $f$ is univalent in $D(0, R)$ and $\partial f(D(0, R))$ has positive hyperbolic curvature. In particular, $f$ is starlike with respect to the origin in $D(0, R)$. This implies that $f(\partial D(0, R))$ is an analytic curve which is starlike with respect to the origin. Therefore, $f$ is injective on $\partial D(0, R)$. But if $f$ is injective on the compact set $\overline{D(0, R)}$, then $f$ must remain injective on a slightly larger disk, which contradicts the definition of $R$. Consequently, we must have $R=1$.

Thus, $f$ is univalent in $\mathbb{D}$ and $f(D(0, r))$ is hyperbolically convex for $0<r<1$. Let $\left\{r_{n}\right\}$ be an increasing sequence of positive numbers tending to 1 . Because an increasing union of hyperbolically convex sets is again 
hyperbolically convex, the set $f(\mathbb{D})=\bigcup\left\{f\left(D\left(0, r_{n}\right)\right): n=1,2,3, \ldots\right\}$ is hyperbolically convex.

Corollary. Let $f$ be holomorphic and univalent in $\mathbb{D}$ with $f(\mathbb{D}) \subset \mathbb{D}$. Then $f$ is hyperbolically convex if and only if $f$ maps every subdisk of $\mathbb{D}$ onto a hyperbolically convex region. In particular, for $0<r<1$ the function $f(r z)$ is hyperbolically convex.

Proof. In the proof of (iii) $\Rightarrow(\mathrm{i})$ we showed that $f(D(0, r))$ is hyperbolically convex whenever $f$ is hyperbolically convex. By making use of the invariance of hyperbolic convexity under Aut $(\mathbb{D})$, we may easily extend this result to any subdisk of $\mathbb{D}$.

Rem ark. This corollary is the hyperbolic analog of a theorem of Study for euclidean convex functions [15]. There is a similar result for spherically convex functions [8].

We next discuss another characterization for hyperbolically convex functions which relates to euclidean starlikeness.

THEOREM 4. Suppose $f$ is holomorphic and locally univalent in $\mathbb{D}$ with $f(\mathbb{D}) \subset \mathbb{D}$. Then the following are equivalent.

(i) $f$ is hyperbolically convex.

(ii) For each $a \in \mathbb{D}$ the function

$$
F_{a}(z)=\frac{f\left(\frac{z+a}{1+\bar{a} z}\right)-f(a)}{1-\overline{f(a)} f\left(\frac{z+a}{1+\bar{a} z}\right)}
$$

is starlike with respect to the origin in $\mathbb{D}$.

(iii) For $(z, a) \in \mathbb{D} \times \mathbb{D}$,

$$
\operatorname{Re}\left\{\frac{(z-a)(1-\bar{a} z) f^{\prime}(z)}{(f(z)-f(a))(1-\overline{f(a)} f(z))}\right\}>0 .
$$

Proof. (i) $\Rightarrow$ (ii). If $f$ is hyperbolically convex, then so is $F_{a}$ for each $a \in \mathbb{D}$ and $F_{a}(0)=0$. Because a hyperbolically convex region in $\mathbb{D}$ which contains the origin is starlike with respect to the origin, the function $F_{a}$ is starlike with respect to the origin.

(ii) $\Rightarrow$ (i). Suppose $F_{a}$ is starlike with respect to the origin for each $a \in \mathbb{D}$. Fix $a, b \in \mathbb{D}$. Then the segment

$$
\left[0, F_{a}((b-a) /(1-\bar{a} b))\right]=[0,(f(b)-f(a)) /(1-\overline{f(a)} f(b))]
$$


lies in $F_{a}(\mathbb{D})$. Note that

$$
f(z)=\frac{F_{a}\left(\frac{z-a}{1-\bar{a} z}\right)+f(a)}{1+\overline{f(a)} F_{a}\left(\frac{z-a}{1-\bar{a} z}\right)} .
$$

Therefore, the conformal automorphism $T(w)=(w+f(a)) /(1+\overline{f(a)} w)$ of $\mathbb{D}$ maps $F_{a}(\mathbb{D})$ onto $f(\mathbb{D})$. Because $T$ also maps $\left[0, F_{a}((b-a) /(1-\bar{a} b))\right]$ onto the hyperbolic geodesic connecting $f(a)$ and $f(b)$, it follows that the hyperbolic geodesic joining $f(a)$ and $f(b)$ lies in $f(\mathbb{D})$. Thus, $f$ is hyperbolically convex.

(ii) $\Leftrightarrow$ (iii). Since the function $F_{a}$ satisfies $F_{a}(0)=0$, it is starlike with respect to the origin if and only if for $z \in \mathbb{D}$

$$
\operatorname{Re}\left\{\frac{z F_{a}^{\prime}(z)}{F_{a}(z)}\right\}>0 .
$$

For $w=(z+a) /(1+\bar{a} z)$ we have

$$
\frac{z F_{a}^{\prime}(z)}{F_{a}(z)}=\frac{\left(1-|f(a)|^{2}\right)(w-a)(1-\bar{a} w) f^{\prime}(w)}{\left(1-|a|^{2}\right)(f(w)-f(a))(1-\overline{f(a)} w)} .
$$

Upon replacing $w$ by $z$, we obtain the desired inequality.

4. Growth and distortion theorems for hyperbolically convex functions. Although the inequality $\left|D_{h 2} f(z) /\left(2 D_{h 1} f(z)\right)\right|<1(z \in \mathbb{D})$ of Theorem 3 characterizes hyperbolically convex functions, it is not a sharp inequality. We now establish the sharp version of Theorem 3(ii) by using a technique of Wirths [16].

TheOREm 5. Let $f$ be hyperbolically convex in $\mathbb{D}$. Then, for $z \in \mathbb{D}$, $\left|D_{h 2} f(z) /\left(2 D_{h 1} f(z)\right)\right| \leq 1-\left|D_{h 1} f(z)\right|^{2}$.

Proof. By considering $f(r z)$ and letting $r$ tend to 1 if necessary, we may assume that $f$ is holomorphic and univalent on $\overline{\mathbb{D}}$ with $|f(z)| \leq R<1$ $(z \in \overline{\mathbb{D}})$. Define

$$
H_{f}(z)=\frac{\left|D_{h 1} f(z)\right|^{2}}{1-\left|D_{h 2} f(z) /\left(2 D_{h 1} f(z)\right)\right|} .
$$

The desired inequality is the same as $H_{f}(z) \leq 1$. Under our assumptions, $H_{f}(z)$ tends to 0 as $|z|$ tends to 1 . If the inequality $H_{f}(z) \leq 1$ were not valid, then there would exist $a \in \mathbb{D}$ such that $H_{f}(a)>1$ and $H_{f}(z) \leq H_{f}(a)$ in a neighborhood of $a$. Without loss of generality we may assume $a=0$. Otherwise, we compose $f$ with an appropriate conformal automorphism of $\mathbb{D}$ and use the invariance properties of hyperbolic convexity and the operators $D_{h 1}$ and $D_{h 2}$. 
Let

$$
f(z)=a_{1} z+a_{2} z^{2}+a_{3} z^{3}+\ldots=a_{1}\left[z+A_{2} z^{2}+A_{3} z^{3}+\ldots\right] .
$$

We are assuming that $H_{f}(z) \leq H_{f}(0)$ in a neighborhood of 0 and

$$
H_{f}(0)=\frac{\left|a_{1}\right|^{2}}{1-\left|A_{2}\right|}>1 .
$$

The inequality $H_{f}(z) \leq H_{f}(0)$ is equivalent to

$$
M_{f}(z) \equiv\left|a_{1}\right|^{2}-\left|a_{1}\right|^{2}\left|D_{h 2} f(z) /\left(2 D_{h 1} f(z)\right)\right|-\left(1-\left|A_{2}\right|\right)\left|D_{h 1} f(z)\right|^{2} \geq 0 .
$$

Direct computation gives

$$
M_{f}(z)=\operatorname{Re}\left\{R_{1} z\right\}+\operatorname{Re}\left\{R_{2} z^{2}\right\}+R_{3}|z|^{2}+O\left(|z|^{3}\right),
$$

where

$$
R_{1}=-\left|a_{1}\right|^{2} \frac{A_{2}}{\left|A_{2}\right|}\left(\frac{3 A_{3} \bar{A}_{2}}{A_{2}}-6\left|A_{2}\right|^{2}+\left|a_{1}\right|^{2}-1+4\left|A_{2}\right|\right)
$$

and

$$
\begin{aligned}
\frac{4\left|A_{2}\right|}{\left|a_{1}\right|^{2}} R_{3}= & \left.\left|\frac{3 A_{3} \bar{A}_{2}}{A_{2}}-2\right| A_{2}\right|^{2}+\left|a_{1}\right|^{2}-\left.1\right|^{2}-2\left|3 A_{3}-2 A_{2}^{2}\right|^{2} \\
& -8\left|a_{1}\right|^{2}\left|A_{2}\right|^{2}+4\left|A_{2}\right|^{2}-2\left(1-\left|a_{1}\right|^{2}\right)^{2}-16\left|A_{2}\right|^{3}\left(1-\left|A_{2}\right|\right) \\
& +8\left(1-\left|a_{1}\right|^{2}\right)\left|A_{2}\right|\left(1-\left|A_{2}\right|\right) .
\end{aligned}
$$

(To aid the reader in verifying the preceding expressions for $R_{1}$ and $R_{3}$, we give a number of intermediate formulas in an appendix.) The fact that $M_{f}(z) \geq 0$ in a neighborhood of 0 yields $R_{1}=0$ and $R_{3} \geq 0$. Thus

from which we see that

$$
\frac{3 A_{3} \bar{A}_{2}}{A_{2}}-6\left|A_{2}\right|^{2}+\left|a_{1}\right|^{2}-1+4\left|A_{2}\right|=0,
$$

$$
\left.\left|\frac{3 A_{3} \bar{A}_{2}}{A_{2}}-2\right| A_{2}\right|^{2}+\left|a_{1}\right|^{2}-\left.1\right|^{2}=16\left|A_{2}\right|^{2}\left(1-\left|A_{2}\right|\right)^{2}
$$

and

$$
\left|3 A_{3}-2 A_{2}^{2}\right|^{2}=\left(4\left|A_{2}\right|^{2}-4\left|A_{2}\right|+1-\left|a_{1}\right|^{2}\right)^{2} .
$$

Furthermore, we have

$$
\frac{4\left|A_{2}\right|}{\left|a_{1}\right|^{2}} R_{3}=-4\left(\left|A_{2}\right|-1+\left|a_{1}\right|^{2}\right)\left(-4\left|A_{2}\right|^{2}+5\left|A_{2}\right|-1+\left|a_{1}\right|^{2}\right) .
$$

Since $H_{f}(0)>1,\left|A_{2}\right|-1+\left|a_{1}\right|^{2}>0$. From Theorem 3 we see that $\left|A_{2}\right|<1$. It is easy to check that $-4\left|A_{2}\right|^{2}+5\left|A_{2}\right|-1+\left|a_{1}\right|^{2}>0$ when $1-\left|a_{1}\right|^{2}<\left|A_{2}\right|<1$. But this contradicts $R_{3} \geq 0$. So we must have $H_{f}(z) \leq 1$ for all $z \in \mathbb{D}$.

Corollary. If $f \in K_{h}(\alpha)$, then $\left|f^{\prime \prime}(0)\right| \leq 2 \alpha\left(1-\alpha^{2}\right)$. Equality holds for $k_{\alpha}$ and its rotations. 
Re mark. For $f$ univalent in $\mathbb{D}$ with $f(\mathbb{D}) \subset \mathbb{D}, f(0)=0$ and $f^{\prime}(0)=$ $\alpha>0$, Pick [13] established the sharp bound $\left|f^{\prime \prime}(0)\right| \leq 4 \alpha(1-\alpha)$; see [2] for a simple proof. The preceding corollary is an improvement of this result for hyperbolically convex functions. The analogous bound for spherically convex functions is given in [11].

Theorem 6. Suppose $f$ is hyperbolically convex in $\mathbb{D}$ and $f \notin \operatorname{Aut}(\mathbb{D})$. Then for any path $\gamma$ in $\mathbb{D}$ from a to $b$,

$$
\begin{aligned}
& \frac{\left|D_{h 1} f(a)\right|}{\sqrt{1-\left|D_{h 1} f(a)\right|^{2}}} \exp \left[-2 \operatorname{length}_{h}(\gamma)\right] \\
& \quad \leq \frac{\left|D_{h 1} f(b)\right|}{\sqrt{1-\left|D_{h 1} f(b)\right|^{2}}} \leq \frac{\left|D_{h 1} f(a)\right|}{\sqrt{1-\left|D_{h 1} f(a)\right|^{2}}} \exp \left[2 \operatorname{length}_{h}(\gamma)\right]
\end{aligned}
$$

where $\operatorname{length}_{h}(\gamma)$ is the hyperbolic length of the path $\gamma$.

P r o of. It suffices to establish the upper bound because the lower bound then follows by interchanging the roles of $a$ and $b$. Let $\gamma: z=z(s), 0 \leq s \leq$ $L=\operatorname{length}_{h}(\gamma)$, be a parametrization of $\gamma$ by hyperbolic arc length. This implies that

$$
z^{\prime}(s)=[1-z(s) \overline{z(s)}] e^{i \theta(s)} .
$$

Direct calculation then results in (see [7])

$$
\frac{(d / d s)\left|D_{h 1} f(z)\right|}{\left|D_{h 1} f(z)\right|}=\frac{d}{d s} \log \left|D_{h 1} f(z)\right|=\operatorname{Re}\left\{\frac{D_{h 2} f(z)}{D_{h 1} f(z)} e^{i \theta(s)}\right\} \leq\left|\frac{D_{h 2} f(z)}{D_{h 1} f(z)}\right| .
$$

Theorem 5 gives

$$
\frac{(d / d s)\left|D_{h 1} f(z)\right|}{\left|D_{h 1} f(z)\right|\left(1-\left|D_{h 1} f(z)\right|^{2}\right)} \leq 2,
$$

since $f \notin \operatorname{Aut}(\mathbb{D})$. By integrating this differential inequality over the interval $[0, L]$, we obtain

$$
\log \frac{\left|D_{h 1} f(b)\right| \sqrt{1-\left|D_{h 1} f(a)\right|^{2}}}{\left|D_{h 1} f(a)\right| \sqrt{1-\left|D_{h 1} f(b)\right|^{2}}} \leq 2 L .
$$

This gives the upper bound in the theorem.

Corollary. For $f \in K_{h}(\alpha)$, we have the sharp bounds

$$
\begin{aligned}
\frac{\alpha(1-|z|)}{\sqrt{(1+|z|)^{2}-4 \alpha^{2}|z|}} & =D_{h 1} k_{\alpha}(-|z|) \leq\left|D_{h 1} f(z)\right| \leq D_{h 1} k_{\alpha}(|z|) \\
& =\frac{\alpha(1+|z|)}{\sqrt{(1-|z|)^{2}+4 \alpha^{2}|z|}}
\end{aligned}
$$

Proof. These inequalities are trivial for $\alpha=1$. If $\alpha \in(0,1)$, then $f$ is not a conformal automorphism of $\mathbb{D}$. To establish the inequalities in this 
case, let $a=0, b=z$ and $\gamma$ be the hyperbolic geodesic from 0 to $z$ in the theorem. Since $D_{h 1} f(0)=\alpha$, the theorem becomes

$$
\frac{\alpha}{\sqrt{1-\alpha^{2}}} \cdot \frac{1-|z|}{1+|z|} \leq \frac{\left|D_{h 1} f(z)\right|}{\sqrt{1-\left|D_{h 1} f(z)\right|^{2}}} \leq \frac{\alpha}{\sqrt{1-\alpha^{2}}} \cdot \frac{1+|z|}{1-|z|} .
$$

Because $t / \sqrt{1-t^{2}}$ is an increasing function on $(0,1)$, the desired inequalities follow from solving the above inequalities for $\left|D_{h 1} f(z)\right|$. All that remains is to calculate $D_{h 1} k_{\alpha}(x)$ for $-1<x<1$, which can be done directly using the expression for $k_{\alpha}$.

TheOrem 7. Let $f \in K_{h}(\alpha)$. Then we have sharp estimates

$$
-k_{\alpha}(-|z|) \leq|f(z)| \leq k_{\alpha}(|z|) .
$$

Proof. To prove the upper bound, fix any $a \in \mathbb{D}$, set $\gamma=[0, a]$ and $\Gamma=f \circ \gamma$. Then from the Corollary to Theorem 6,

$$
\begin{aligned}
d_{\mathbb{D}}(0, f(a)) & \leq \int_{\Gamma} \frac{|d w|}{1-|w|^{2}}=\int_{\gamma} \frac{\left|f^{\prime}(z)\right||d z|}{1-|f(z)|^{2}}=\int_{\gamma}\left|D_{h 1} f(z)\right| \frac{|d z|}{1-|z|^{2}} \\
& \leq \int_{\gamma} D_{h 1} k_{\alpha}(|z|) \frac{|d z|}{1-|z|^{2}}=\int_{0}^{|z|} D_{h 1} k_{\alpha}(t) \frac{d t}{1-t^{2}} \\
& =\int_{0}^{|z|} \frac{k_{\alpha}^{\prime}(t) d t}{1-k_{\alpha}(t)^{2}}=d_{\mathbb{D}}\left(0, k_{\alpha}(|z|)\right) .
\end{aligned}
$$

This is equivalent to the upper bound.

In order to establish the lower bound, fix $r=|z|, 0<r<1$, and assume $f(a)$ is the nearest point to the origin on $f(\{z:|z|=r\})$. Set $\Gamma=[0, f(a)]$ and $\gamma=f^{-1} \circ \Gamma$. By using the Corollary to Theorem 6, we have

$$
\begin{aligned}
d_{\mathbb{D}}(0, f(a)) & =\int_{\Gamma} \frac{|d w|}{1-|w|^{2}}=\int_{\gamma} \frac{\left|f^{\prime}(z)\right||d z|}{1-|f(z)|^{2}}=\int_{\gamma}\left|D_{h 1} f(z)\right| \frac{|d z|}{1-|z|^{2}} \\
& \geq \int_{\gamma} D_{h 1} k_{\alpha}(-|z|) \frac{d|z|}{1-|z|^{2}}=\int_{0}^{|z|} D_{h 1} k_{\alpha}(-t) \frac{d t}{1-(-t)^{2}} \\
& =\int_{0}^{|z|} \frac{k_{\alpha}^{\prime}(-t) d t}{1-k_{\alpha}(-t)^{2}}=d_{\mathbb{D}}\left(0,-k_{\alpha}(-|z|)\right) .
\end{aligned}
$$

The inequalities $d_{\mathbb{D}}(0, f(z)) \geq d_{\mathbb{D}}(0, f(a)) \geq d_{\mathbb{D}}(0,-k(-|z|))$ then imply the desired lower bound.

5. The radius of hyperbolic convexity. For the class $S B(\alpha)$ we shall determine the sharp radius of hyperbolic convexity. In fact, it is no more 
difficult to determine the sharp radius of hyperbolic $k$-convexity for $k \in$ $[0,4]$, so we do this.

Example 2. The function

$$
K_{\alpha}(z)=\frac{4 \alpha z}{\left[\sqrt{(1-z)^{2}+4 \alpha z}+1-z\right]^{2}}
$$

belongs to $S B(\alpha)$ and satisfies the functional relationship

$$
\left(\frac{1+K_{\alpha}(z)}{1-K_{\alpha}(z)}\right)^{2}-1=\alpha\left[\left(\frac{1+z}{1-z}\right)^{2}-1\right] .
$$

The image $K_{\alpha}(\mathbb{D})$ is the unit disk with the segment

$$
\left(-1, \frac{-\alpha}{2-\alpha+2 \sqrt{1-\alpha}}\right]
$$

removed. The function $K_{\alpha}$ is extremal for several problems over the class $S B(\alpha)$. For example, if $F \in S B(\alpha)$, then

$$
\left|F^{\prime \prime}(0)\right| \leq 4 \alpha(1-\alpha)=4\left|F^{\prime}(0)\right|\left(1-\left|F^{\prime}(0)\right|\right)
$$

with equality if and only if $F$ is a rotation of $K_{\alpha}$ [13]. This is formulated in invariant terms as

$$
\left|D_{h 2} f(z)\right| \leq 4\left|D_{h 1} f(z)\right|\left(1-\left|D_{h 1} f(z)\right|\right)
$$

for any univalent function $f$ mapping $\mathbb{D}$ into itself. Direct calculation using the functional equation for $K_{\alpha}$ yields

$$
D_{h 2} K_{\alpha}(x)=4 D_{h 1} K_{\alpha}(x)\left(1-D_{h 1} K_{\alpha}(x)\right)
$$

for $x \in(-1,1)$. Another sharp result is that for $F \in S B(\alpha)$,

$$
\frac{\alpha(1-|z|)^{2}}{(1+|z|)^{2}-4 \alpha|z|} \leq\left|D_{h 1} F(z)\right| \leq \frac{\alpha(1+|z|)^{2}}{(1-|z|)^{2}+4 \alpha|z|}
$$

with equality if and only if $F$ is a rotation of $K_{\alpha}([7],[13])$.

THEOREM 8. For $k \in[0,4]$ the radius of hyperbolic $k$-convexity for the family $S B(\alpha)$ is the unique root $r(\alpha, k)$ in the interval $(0,1]$ of the polynomial

$$
p_{\alpha, k}(r)=r^{4}-(2+\alpha k) r^{3}+(8 \alpha+2 \alpha k-6) r^{2}-(2+\alpha k) r+1 .
$$

The root $r(\alpha, k)$ is an increasing function of $\alpha$ which satisfies the sharp bounds

$$
2-\sqrt{3} \leq r(\alpha, k) \leq \begin{cases}1 & \text { if } 0 \leq k \leq 2 \\ {\left[k-\sqrt{k^{2}-4}\right] / 2} & \text { if } 2 \leq k \leq 4\end{cases}
$$

In particular, $r(\alpha, 4)$ is the constant $2-\sqrt{3}$, independent of $\alpha$.

Proof. Note that if $\gamma: z(t)=r e^{i t}$ is the positively oriented circle of radius $r \in(0,1)$, then $\kappa_{\mathbb{D}}(z, \gamma)=r+r^{-1}$. By formula (2) we wish to 
determine the maximal $r$ such that

$$
\kappa_{\mathbb{D}}(z, \gamma)+\operatorname{Im}\left\{\frac{D_{h 2} F(z)}{D_{h 1} F(z)} \cdot \frac{z^{\prime}(t)}{\left|z^{\prime}(t)\right|}\right\} \geq k\left|D_{h 1} F(z)\right|, \quad z \in \gamma .
$$

This inequality holds if

$$
\frac{r^{2}+1}{r}-\left|\frac{D_{h 2} F(z)}{D_{h 1} F(z)}\right| \geq k\left|D_{h 1} F(z)\right|, \quad z \in \gamma .
$$

By making use of inequality (3), this will hold if

$$
\frac{r^{2}+1}{r}-4+(4-k)\left|D_{h 1} F(z)\right| \geq 0, \quad z \in \gamma .
$$

Because $k \in[0,4]$, the lower bound in inequality (4) implies that the desired inequality will hold if

$$
\frac{r^{2}+1}{r}-4+\frac{(4-k) \alpha(1-r)^{2}}{(1+r)^{2}-4 \alpha r} \geq 0
$$

The function $\left(r^{2}+1\right) / r-4$ decreases strictly from $\infty$ to -2 on $[0,1]$ while the function $\alpha(1-r)^{2} /\left[(1+r)^{2}-4 \alpha r\right]$ decreases strictly from $\alpha$ to 0 on $[0,1]$. Therefore the graphs of $\left(r^{2}+1\right) / r-4$ and $(k-4) \alpha(1-r)^{2} /\left[(1+r)^{2}-4 \alpha r\right]$ cross at a unique point in $(0,1]$. The numerator of the rational function given by the left-hand side of inequality $(5)$ is $p_{\alpha, k}(r)$.

Now we establish the sharpness. For the function $K_{\alpha}$ and the path $\gamma$ : $z(t)=r e^{i t}, 0 \leq t \leq 2 \pi$, the choice $t=-\pi$ in formula (2) results in

$$
\begin{aligned}
\kappa_{\mathbb{D}}\left(K_{\alpha}(-r)\right. & \left., K_{\alpha} \circ \gamma\right)\left|D_{h 1} K_{\alpha}(-r)\right| \\
& =\kappa_{\mathbb{D}}(-r, \gamma)+\operatorname{Im}\left\{\frac{D_{h 2} K_{\alpha}(-r)}{D_{h 1} K_{\alpha}(-r)}(-i)\right\} \\
& =\frac{1+r^{2}}{r}-\frac{D_{h 2} K_{\alpha}(-r)}{D_{h 1} K_{\alpha}(-r)}=\frac{1+r^{2}}{r}-\left[4\left(1-D_{h 1} K_{\alpha}(-r)\right)\right] \\
& =\frac{1+r^{2}}{r}-4+(4-k) D_{h 1} K_{\alpha}(-r)+k D_{h 1} K_{\alpha}(-r) \\
& =\frac{r^{2}+1}{r}-4+\frac{(4-k) \alpha(1-r)^{2}}{(1+r)^{2}-4 \alpha r}+k D_{h 1} K_{\alpha}(-r) .
\end{aligned}
$$

The choice $r=r(\alpha, k)$ produces $\kappa_{\mathbb{D}}\left(K_{\alpha}(-r), K_{\alpha} \circ \gamma\right)=k$, while $\kappa_{\mathbb{D}}\left(K_{\alpha}(-r)\right.$, $\left.K_{\alpha} \circ \gamma\right)<k$ for $r(\alpha, k)<r<1$.

From inequality (5) and the discussion immediately following it, we conclude that for each fixed $k \in[0,4]$, the value of $r(\alpha, k)$ is a continuous, increasing function of $\alpha$ on the interval $(0,1]$; in fact, we can view $r(\alpha, k)$ as continuous on the interval $[0,1]$. Note that

$$
p_{0, k}(r)=r^{4}-2 r^{3}-6 r^{2}-2 r+1=(r+1)^{2}\left(r^{2}-4 r+1\right) .
$$


This reveals that $r(0, k)=2-\sqrt{3}$. Consequently, $r(\alpha, k) \geq 2-\sqrt{3}$ for $\alpha \in(0,1]$. Next,

$p_{1, k}(r)=r^{4}-(2+k) r^{3}+(2+2 k) r^{2}-(2+k) r+1=(r-1)^{2}\left(r^{2}-k r+1\right)$.

This shows that $r(1, k)=1$ for $0 \leq k \leq 2$, while $r(1, k)=\left[k-\sqrt{k^{2}-4}\right] / 2$ for $2 \leq k \leq 4$. For $k=4$ we now conclude that $r(\alpha, 4)=2-\sqrt{3}$ for all $\alpha \in(0,1]$.

6. An alternate definition of hyperbolic linear invariance. Pommerenke [14] formally started the study of (euclidean) linearly invariant families of holomorphic functions. Pommerenke's definition of linear invariance was given a purely geometric interpretation in [6]. In [7] the authors proposed a definition of hyperbolic linear invariance for locally univalent functions $f: \mathbb{D} \rightarrow \mathbb{D}$ and in $[8]$ they defined spherical linear invariance for locally univalent meromorphic functions $f: \mathbb{D} \rightarrow \mathbb{P}$, where $\mathbb{P}$ denotes the Riemann sphere. The definitions of euclidean and spherical linear invariance are quite similar, while the definition of hyperbolic linear invariance in [7] is different. One reason for the difference is that the definition of hyperbolic linear invariance proposed in [7] was motivated by the desire to generalize parts of the theory of bounded univalent function theory in the same way that Pommerenke's definition of (euclidean) linear invariance extended aspects of the theory of univalent functions. We now offer a second definition for the hyperbolic linearly invariant order of a locally univalent function $f: \mathbb{D} \rightarrow \mathbb{D}$. This new definition directly parallels those of euclidean and spherical linear invariance; it is now a viable alternative because we have developed the theory of hyperbolically convex functions. But this new definition does not seem to extend parts of the classical theory of bounded univalent functions in the way that the former definition did.

For a locally univalent function $f: \mathbb{D} \rightarrow \mathbb{D}$, set

$$
\widetilde{\alpha}_{h}(f)=\sup \left\{\left|\frac{D_{h 2} f(z)}{2 D_{h 1} f(z)}\right|: z \in \mathbb{D}\right\} .
$$

We shall see that this new definition of hyperbolic linear invariance leads to a number of results analogous to those for euclidean and spherical linear invariance. In fact, the proofs of the analogous results are so similar that we typically omit all details. In [7] we defined the hyperbolic linearly invariant order by

$$
\alpha_{h}(f)=\sup \left\{\left|\frac{D_{h 2} f(z)}{2 D_{h 1} f(z)\left(1-\left|D_{h 1} f(z)\right|\right)}\right|: z \in \mathbb{D}\right\} .
$$

Clearly, $\widetilde{\alpha}_{h}(f) \leq \alpha_{h}(f)$. For $\alpha \geq 1$, let $\widetilde{\mathcal{F}}(\alpha)$ denote the family of all $f$ with $\widetilde{\alpha}_{h}(f) \leq \alpha$. Pick's sharp upper bound on $\left|f^{\prime \prime}(0)\right|$ for $f \in S B(\alpha)$ implies that $\widetilde{\mathcal{F}}(2)$ contains all univalent functions $f$ that map $\mathbb{D}$ into itself. 
We introduce other quantities related to hyperbolic linear invariance. Define $\varrho_{h}(f)$, the hyperbolic radius of uniform local hyperbolic convexity, for a locally univalent function $f: \mathbb{D} \rightarrow \mathbb{D}$ by

$\sup \left\{\varrho: f\right.$ is univalent in $D_{\mathbb{D}}(a, \varrho)$ and

$$
\left.f\left(D_{\mathbb{D}}(a, \varrho)\right) \text { is hyperbolically convex for all } a \in \mathbb{D}\right\} \text {. }
$$

We say that $f$ is uniformly locally hyperbolically convex if $\varrho_{h}(f)>0$. Let $C_{h}(\varrho)$ be the family of uniformly locally hyperbolically convex functions with $\varrho_{h}(f) \geq \varrho$. Define $k_{h}(f)$ by

$\inf \{k \geq 2: f(\Omega)$ is hyperbolically convex for each hyperbolically $k$-convex $\Omega \subset \mathbb{D}\}$.

Later we will see why we can restrict $k \geq 2$ with no essential loss of generality.

In the case of euclidean (spherical) linear invariance it is known that the euclidean (spherical) linearly invariant order is at least one and equals one if and only if the function is univalent and euclidean (spherically) convex. The analogous result does not hold for the definition of the hyperbolic linearly invariant order $\widetilde{\alpha}_{h}(f)$ proposed above.

ExAMPLE 3. For a conformal automorphism $T$ of $\mathbb{D}$ we will show that $\widetilde{\alpha}_{h}(T)=0$. If $T \in \operatorname{Aut}(\mathbb{D})$, then $\left|D_{h 1} T\right|=1$, so it suffices to show $\mathbb{D}_{h 2} T=0$. Fix $a \in \mathbb{D}$. Let

$$
R(z)=\frac{z+a}{1+\bar{a} z} \quad \text { and } \quad S(w)=\frac{w-T(a)}{1-\overline{T(a)} w} .
$$

Then $F=S \circ T \circ R \in \operatorname{Aut}(\mathbb{D})$ and fixes the origin, so $F(z)=\lambda z$ for some unimodular constant $\lambda$. Hence, $D_{h 2} T(a)=F^{\prime \prime}(0)=0$.

However, the next result shows that the proposed hyperbolic linearly invariant order $\widetilde{\alpha}_{h}(f)$ almost behaves as in the euclidean and spherical cases.

Theorem 9. Suppose $f: \mathbb{D} \rightarrow \mathbb{D}$ is locally univalent. If $\widetilde{\alpha}_{h}(f)<1$, then $f \in \operatorname{Aut}(\mathbb{D})$. For $f \notin \operatorname{Aut}(\mathbb{D}), \widetilde{\alpha}_{h}(f) \geq 1$ with equality if $f$ is hyperbolically convex.

Pro of. Let $\alpha=\widetilde{\alpha}_{h}(f)$. We begin by showing that for $z \in \mathbb{D}$,

$$
\frac{\left|f^{\prime}(0)\right|}{1-|f(0)|^{2}} \cdot \frac{(1-|z|)^{\alpha-1}}{(1+|z|)^{\alpha+1}} \leq \frac{\left|f^{\prime}(z)\right|}{1-|f(z)|^{2}}
$$

Let $z=z(s), 0 \leq s \leq L$, be a parametrization of the radial segment $\gamma=[0, z]$ by hyperbolic arc length. Then as in the proof of Theorem 6 we obtain

$$
\frac{(d / d s)\left|D_{h 1} f(z)\right|}{\left|D_{h 1} f(z)\right|}=\operatorname{Re}\left\{\frac{d}{d s} \log D_{h 1} f(z)\right\}=\operatorname{Re}\left\{\frac{D_{h 2} f(z)}{D_{h 1} f(z)} e^{i \theta(s)}\right\} \geq-2 \alpha .
$$


By integrating this inequality with respect to $s$ over $\gamma$, we get

$$
\log \frac{\left|D_{h 1} f(z)\right|}{\left|D_{h 1} f(0)\right|} \geq-2 \alpha d_{\mathbb{D}}(0, z)=-\alpha \log \left(\frac{1+|z|}{1-|z|}\right),
$$

which is equivalent to inequality (6).

If $\alpha<1$, then from inequality (6) it follows that

$$
\lim _{|z| \rightarrow 1} \frac{\left|f^{\prime}(z)\right|}{1-|f(z)|^{2}}=\infty .
$$

Now, $\lambda_{R}(z)|d z|=R|d z| /\left(R^{2}-|z|^{2}\right)$ is the hyperbolic metric on $D(0, R)$ and so has Gaussian curvature -4 . Also, $\mu(z)|d z|=\left|f^{\prime}(z)\right||d z| /\left(1-|f(z)|^{2}\right)$ has Gaussian curvature -4 on $\mathbb{D}$ since it is the pull-back of the hyperbolic metric $\lambda_{\mathbb{D}}(z)|d z|$ via the locally univalent function $f$. For $R>1$, we have

$$
\lim _{|z| \rightarrow 1} \frac{\lambda_{R}(z)}{\mu(z)}=0 .
$$

From $\left[4\right.$, Theorem 2.1] we conclude that $\lambda_{R}(z) \leq \mu(z)$ for $z \in \mathbb{D}$. By letting $R$ decrease to 1 , for $z \in \mathbb{D}$ we obtain

$$
\frac{1}{1-|z|^{2}} \leq \frac{\left|f^{\prime}(z)\right|}{1-|f(z)|^{2}}
$$

The invariant form of Schwarz' Lemma yields the reverse inequality. Therefore, equality holds throughout $\mathbb{D}$ and $f \in \operatorname{Aut}(\mathbb{D})$ by the case of equality in the invariant form of Schwarz' Lemma.

For $f \notin \operatorname{Aut}(\mathbb{D})$, it now follows that $\widetilde{\alpha}_{h}(f) \geq 1$. From Theorem 3 we obtain $\widetilde{\alpha}_{h}(f) \leq 1$ if $f$ is hyperbolically convex. Thus, $\widetilde{\alpha}_{h}(f)=1$ if $f$ is hyperbolically convex.

R e m a r k. From Theorem 9 and formula (2), we can show that there are no locally univalent functions $f: \mathbb{D} \rightarrow \mathbb{D}$ except conformal automorphisms of $\mathbb{D}$ that map every hyperbolically $k$-convex subset $(0 \leq k<2)$ of $\mathbb{D}$ onto a hyperbolically convex region. See $[6$, Lemma] for the completely analogous proof in the euclidean case.

Theorem 10. Suppose $f: \mathbb{D} \rightarrow \mathbb{D}$ is locally univalent and $f \notin \operatorname{Aut}(\mathbb{D})$. Then $\varrho_{h}(f)=\varrho$ if and only if $\widetilde{\alpha}_{h}(f)=\operatorname{coth}(2 \varrho)$. In particular, $C_{h}(\varrho)=$ $\widetilde{\mathcal{F}}(\operatorname{coth}(2 \varrho))$.

Proof. A geometric proof completely parallel to that of [6, Theorem 3] is easily constructed. Note that the condition $\widetilde{\alpha}_{h}(f) \geq 1$ is needed in the proof.

Corollary. If $f: \mathbb{D} \rightarrow \mathbb{D}$ is locally univalent and $\widetilde{\alpha}_{h}(f)=1$, then $f$ is hyperbolically convex. 
TheOREm 11. Suppose $f: \mathbb{D} \rightarrow \mathbb{D}$ is locally univalent. Then $k_{h}(f)=$ $2 \widetilde{\alpha}_{h}(f)$.

Proof. A geometric proof parallel to $[8$, Theorem 9] is readily given.

7. Open problems. For the family $K_{h}(\alpha)$ it would be interesting to obtain the sharp upper bound on $\left|f^{(n)}(0)\right|$ for $n \geq 3$ and on the Schwarzian derivative. For $\alpha=1 / \sqrt{2}$ we note that $k_{1 / \sqrt{2}}$ cannot be the extremal function for the sharp upper bound on $\left|f^{(3)}(0)\right|$ because $k_{1 / \sqrt{2}}^{(3)}(0)=0$. What are sharp upper and lower bounds on $\left|f^{\prime}(z)\right|$ for the family $K_{h}(\alpha)$ ? Also, what are sharp growth, distortion and covering theorems for the family $\widetilde{\mathcal{F}}(\alpha)$ ?

Appendix. The following six expressions are useful in computing $R_{1}$ and $R_{3}$ :

$$
\begin{aligned}
\left(1-|z|^{2}\right) \frac{f^{\prime \prime}(z)}{2 f^{\prime}(z)}= & A_{2}+\left(3 A_{3}-2 A_{2}^{2}\right) z+\left(6 A_{4}-9 A_{3} A_{2}+4 A_{2}^{3}\right) z^{2}-A_{2}|z|^{2}+O\left(z^{3}\right) . \\
\frac{\left(1-|z|^{2}\right) \overline{f(z)} f^{\prime}(z)}{1-|f(z)|^{2}}=\left|a_{1}\right|^{2} \bar{z}+\left|a_{1}\right|^{2} \bar{A}_{2} \bar{z}^{2}+2\left|a_{1}\right|^{2} A_{2}|z|^{2}+O\left(z^{3}\right) . & \\
\frac{D_{h 2} f(z)}{2 D_{h 1} f(z)}= & A_{2}+\left(3 A_{3}-2 A_{2}^{2}\right) z+\left(\left|a_{1}\right|^{2}-1\right) \bar{z}+\left(6 A_{4}-9 A_{2} A_{3}+4 A_{2}^{3}\right) z^{2} \\
& +\left|a_{1}\right|^{2} \bar{A}_{2} \bar{z}^{2}+A_{2}\left(2\left|a_{1}\right|^{2}-1\right)|z|^{2}+O\left(z^{3}\right) . \\
\left|\frac{D_{h 2} f(z)}{2 D_{h 1} f(z) \mid=}\right| & \left|A_{2}\right|+\frac{1}{\left|A_{2}\right|} \operatorname{Re}\left\{\left(3 A_{3} \bar{A}_{2}-2\left|A_{2}\right|^{2} A_{2}+\left|a_{1}\right|^{2} A_{2}-A_{2}\right) z\right\} \\
& +\operatorname{Re}\left\{\frac { 1 } { | A _ { 2 } | } \left[6 A_{4} \bar{A}_{2}-9 A_{3}\left|A_{2}\right|^{2}+4\left|A_{2}\right|^{2} A_{2}^{2}\right.\right. \\
& \left.\left.+\left|a_{1}\right|^{2} A_{2}^{2}+\left(\left|a_{1}\right|^{2}-1\right)\left(3 A_{3}-2 A_{2}^{2}\right)\right] z^{2}\right\} \\
& -\operatorname{Re}\left\{\frac{1}{4\left|A_{2}\right|^{3}}\left[3 A_{3} \bar{A}_{2}-2\left|A_{2}\right|^{2} A_{2}+\left|a_{1}\right|^{2} A_{2}-A_{2}\right]^{2} z^{2}\right\} \\
& +\frac{1}{2\left|A_{2}\right|}\left[4\left|a_{1}\right|^{2} A_{2}^{2}-2\left|A_{2}\right|^{2}+\left|3 A_{3}-2 A_{2}^{2}\right|^{2}-\left(1-\left|a_{1}\right|^{2}\right)^{2}\right]|z|^{2} \\
& -\left.\frac{1}{4\left|A_{2}\right|^{3}}\left|3 A_{3} \bar{A}_{2}-2\right| A_{2}\right|^{2} A_{2}+\left|a_{1}\right|^{2} A_{2}-\left.A_{2}\right|^{2}|z|^{2}+O\left(z^{3}\right) . \\
D_{h 1} f(z)= & a_{1}\left[1+2 A_{2} z+3 A_{3} z^{2}-\left(1-\left|a_{1}\right|^{2}\right)|z|^{2}\right]+O\left(z^{3}\right) . \\
\left|D_{h 1} f(z)\right|^{2}= & \left|a_{1}\right|^{2}\left[1+4 \operatorname{Re}\left\{A_{2} z\right\}+6 \operatorname{Re}\left\{A_{3} z^{2}\right\}\right. \\
& \left.+\left(4\left|A_{1}\right|^{2}-2\left(1-\left|a_{1}\right|^{2}\right)\right)|z|^{2}\right]+O\left(z^{3}\right) .
\end{aligned}
$$




\section{References}

[1] P. Duren, Univalent Functions, Grundlehren Math. Wiss. 259, Springer, New York, 1983.

[2] B. Flinn and B. Osgood, Hyperbolic curvature and conformal mapping, Bull. London Math. Soc. 18 (1986), 272-276.

[3] A. W. Goodman, Univalent Functions, Vols. I and II, Mariner, Tampa, 1983.

[4] M. Heins, On a class of conformal metrics, Nagoya Math. J. 21 (1962), 1-60.

[5] W. Ma, D. Mejia and D. Minda, Distortion theorems for hyperbolically and spherically $k$-convex functions, in: Proc. Internat. Conf. on New Trends in Geometric Function Theory and Applications, R. Parvatham and S. Ponnusamy (eds.), World Sci., Singapore, 1991, 46-54

[6] W. Ma and D. Minda, Euclidean linear invariance and uniform local convexity, J. Austral. Math. Soc. Ser. A 52 (1992), 401-418

[7] - - - Hyperbolic linear invariance and hyperbolic k-convexity, ibid., to appear.

[8] —, -, Spherical linear invariance and uniform local spherical convexity, in: Current Topics in Geometric Function Theory, H. M. Srivastava and S. Owa (eds.), World Sci., Singapore, 1993, 148-170.

[9] D. Mejia and D. Minda, Hyperbolic geometry in hyperbolically $k$-convex regions, Rev. Colombiana Mat. 25 (1991), 123-142.

[10] D. Minda, A reflection principle for the hyperbolic metric and applications to geometric function theory, Complex Variables Theory Appl. 8 (1987), 129-144.

[11] -, Applications of hyperbolic convexity to euclidean and spherical convexity, J. Analyse Math. 49 (1987), 90-105.

[12] B. Osgood, Some properties of $f^{\prime \prime} / f^{\prime}$ and the Poincaré metric, Indiana Univ. Math. J. 31 (1982), 449-461.

[13] G. Pick, Über die konforme Abbildung eines Kreises auf eines schlichtes und zugleich beschränktes Gebiete, S.-B. Kaiserl. Akad. Wiss. Wien 126 (1917), 247-263.

[14] Ch. Pommerenke, Linear-invariante Familien analytischer Funktionen I, Math. Ann. 155 (1964), 108-154

[15] E. Study, Konforme Abbildung einfachzusammenhängender Bereiche, Vorlesungen über ausgewählte Gegenstände der Geometrie, Heft 2, Teubner, Leipzig und Berlin, 1913.

[16] K.-J. Wirths, Coefficient bounds for convex functions of bounded type, Proc. Amer. Math. Soc. 103 (1988), 525-530.

DEPARTMENT OF MATHEMATICAL SCIENCES

UNIVERSITY OF CINCINNATI

CINCINNATI, OHIO 45221-0025

U.S.A. 